\title{
DYNAMO Survey: An Upclose View of Clumpy Galaxies
}

\author{
David Fisher $^{1}$ and the DYNAMO ${ }^{2}$ Team \\ Centre for Astrophysics and Supercomputing, Swinburne University of Technology, P. O. Box \\ 218, Hawthorn, VIC 3122, Australia \\ email: dfisher@swin.edu.au \\ ${ }^{2}$ http://adsabs.harvard.edu/abs/2014MNRAS.437.1070G
}

\begin{abstract}
We highlight recent results on the DYNAMO survey of turbulent, clumpy disks galaxies found at $\mathrm{z}=0.1$. Bright star forming DYNAMO galaxies are found to be very similar in properties to star forming galaxies in the high redshift Universe. Typical star formation rates of turbulent DYNAMO galaxies range $10-80 M_{\odot} \mathrm{yr}^{-1}$. Roughly $2 / 3$ of DYNAMO galaxies have $\mathrm{H} \alpha$ kinematics that are consistent with rotation. The typical gas velocity dispersion of DYNAMO galaxies is $\sigma_{H \alpha} \sim 20-60 \mathrm{~km} \mathrm{~s}^{-1}$. We show that, when convolved to the same resolution, maps of $\mathrm{H} \alpha$ emission in DYNAMO galaxies have essentially identical morphology as that of $z \sim 1-3$ galaxies. Finally, DYNAMO galaxies have high molecular gas fractions $f_{m o l} \sim 20-35 \%$. We note that DYNAMO galaxies are not dwarfs, typical masses are $M_{\text {star }} \sim 0.8-8 \times 10^{10} \mathrm{M}_{\odot}$. These data are all consistent with a scenario in which despite being at relatively low redshift the DYNAMO galaxies are forming stars similarly to that observed in the high-redshift Universe, that is to say star formation is occurring in very massive $\left(M_{\text {clump }} \sim 10^{9} \mathrm{M}_{\odot}\right)$, very large $\left(r_{\text {clump }} \sim 300 \mathrm{pc}\right)$ clumps of gas.
\end{abstract}

Keywords. galaxies: elliptical and lenticular, cD - galaxies: evolution - galaxies: formation

\section{Introduction}

Over $80 \%$ of the stars in our universe formed during an epoch at redshifts $z=1-3$ (Hopkins \& Beacom 2006). Star forming galaxies during this epoch have irregular, clumpy morphologies (Abraham et al. 1996), are very gas rich (Tacconi et al. 2013), and that gas is very turbulent (Genzel et al. 2011). The clumps in these galaxies are super-giant (300$1000 \mathrm{pc}$ ) star-forming complexes, which are an order of magnitude larger than similar regions in local galaxies (Wisnioski et al. 2012). Clump masses are often interpreted as a response to the turbulent ISM containing them, high random motions lead to large Jeans' masses, roughly consistent with observed clump masses at $z \sim 1-3$ (Elmegreen et al. 2008).

Because they are typically observed at $z>1$, clumps are mostly unresolved (Jones et al. 2010), even with HST or AO observations. The picture of how star formation and hence galaxy evolution proceeds during the period when most of the stars were forming is therefore incomplete. A detailed description of the size, density, and kinematics resolving individual clumps in turbulent disks is therefore needed.

\section{DYNAMO Sample}

The DYNAMO sample is a set of nearby galaxies that have strikingly similar kinematics, star formation properties and gas content as high redshift clumpy disk galaxies. The survey includes AAT and $2.5 \mathrm{~m}$ integral field observations of $\mathrm{H} \alpha$ of 95 spiral galaxies with the highest $\mathrm{H} \alpha$ luminosity from SDSS (excluding AGNs). 
DYNAMO $\mathrm{H} \alpha$ maps convolved to $z \sim 2$ resolution

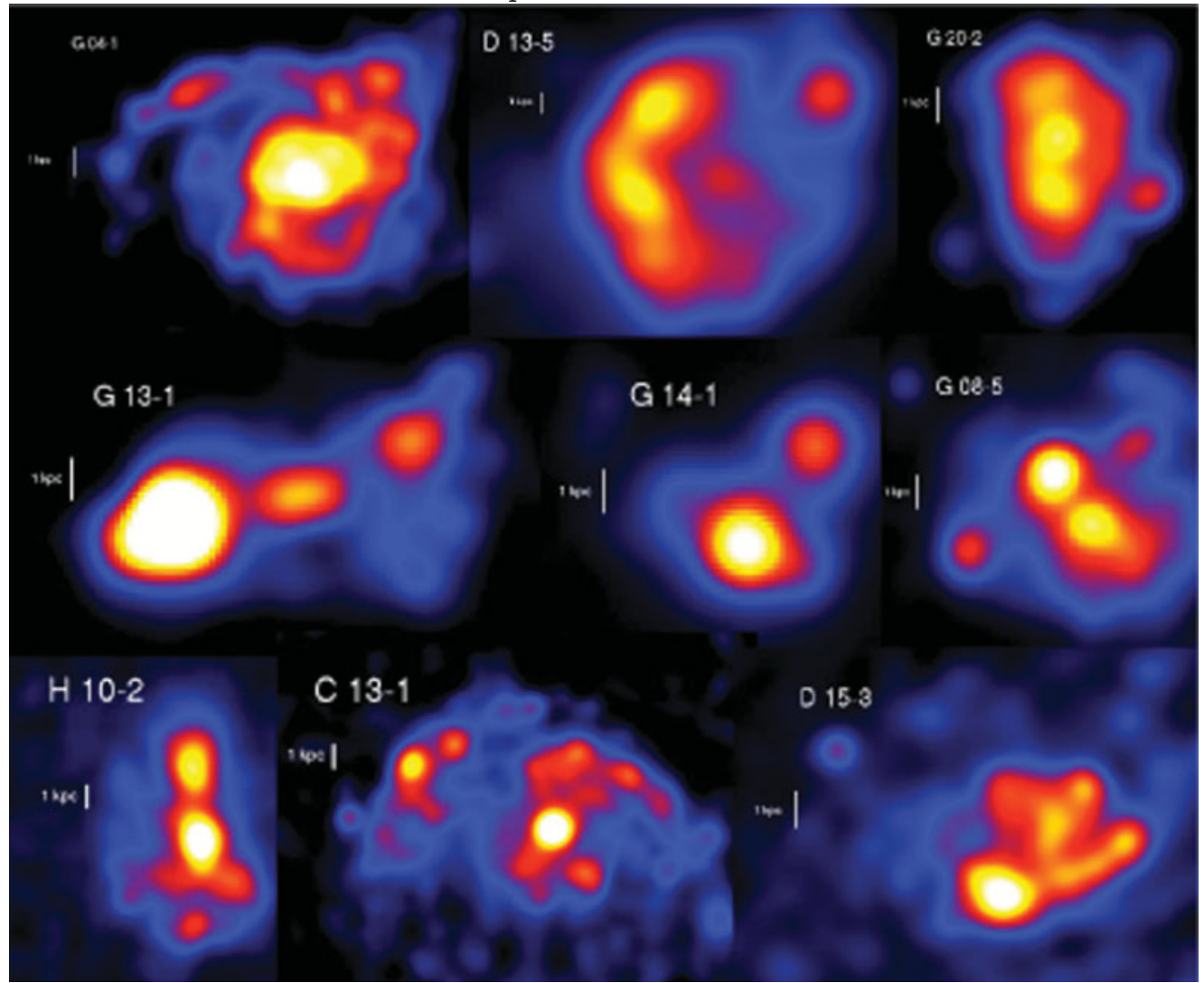

\section{SINS H $\alpha$ maps}

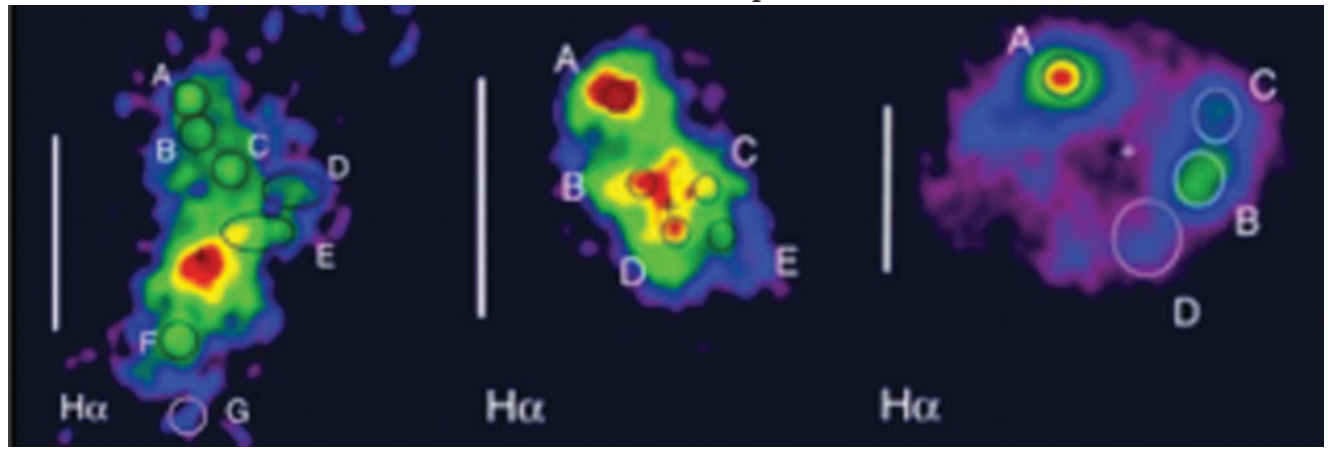

Figure 1. The top 9 panels show HST ACS/WFC maps of $\mathrm{H} \alpha$ emission from the DYNAMO survey of galaxies. The maps have been convolved with a Gaussian that sets the physical resolution to $\sim 800 \mathrm{pc}$. The galaxies are therefore at a similar resolution to how they would appear if they were located at $z \sim 2$. In the bottom panel we show maps from the SINS survey of galaxies (Genzel et al. 2014). When viewed at the same resolution, DYNAMO galaxies appear indistinguishable in morphology to the clumpy, turbulent galaxies of the distant Universe.

DYNAMO galaxies are indeed very good analogues of clumpy $z \sim 2$ galaxies. In Fig. 1 we show $\mathrm{H} \alpha$ maps of DYNAMO galaxies taken with the Hubble Space Telescope ACS/WFC narrow band filters. The maps have been convolved with a Gaussian whose full width at half maximum corresponds to $850 \mathrm{pc}$. These maps, therefore have similar 


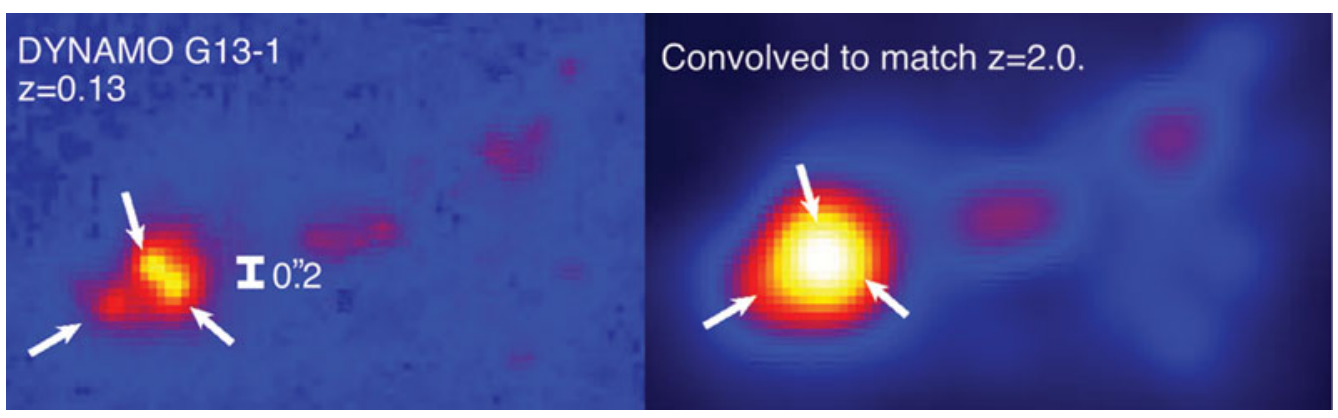

Figure 2. Left panel: HST H $\alpha$ map of, target galaxy DYNAMO G13-1, at the full resolution of HST $(\sim 250$ pc resolution). The white bar shows 0.2 arcsec. Right panel: Simulating the same object as if it were located at $z=2$. Arrows in both maps indicate the location of 3 clumps, identified in the unblurred map. In the $z=2$ map the 3 clumps would appear as one large clump, possibly leading to specious conclusions.

resolution to $\mathrm{H} \alpha$ maps targeting galaxies in $z \sim 1-3$ Universe. We also show three H $\alpha$ maps taken from Genzel et al. (2011) for comparison. When convolved to the same resolution, similarity between DYNAMO galaxies and high- $z$ is quite remarkable. For example, star forming rings are common in high-z clumpy galaxies (e.g. Genzel et al. 2014). Indeed we detect ring-like distributions of $\mathrm{H} \alpha$ emission in a number of DYNAMO galaxies, most prominently in G04-1, D13-5 and G20-2 (top row in Fig. 1). We also detect chains of star forming clumps (G13-1, H10-2). 'Chain galaxies' have been known about in the high redshift Universe for many years, and have been shown in $\mathrm{H} \alpha$ maps of $z \sim 1-3$ galaxies by a number of surveys (e.g. Genzel et al. 2011; Wisnioski et al. 2012; Swinbank et al. 2012).

In Fig 2 we compare the morphology of clumps at resolution set to match a $z=2$ galaxy, to the full resolution of a DYNAMO galaxy. It is clear that the factor of $\sim 3 \times$ finer spatial resolution of a galaxy at $z=0.1$ significantly affects the identification of clumps. In the low-resolution $\mathrm{H} \alpha$ maps, set to match observations of a $z \sim 2$ galaxy, the ionized gas is arranged into 3 clumps that are fairly large. At the full HST resolution of a $z=0.14$ DYNAMO galaxy (shown left in Fig 2) the clumps of ionized gas emission are smaller, and those clumps in the low resolution map. The typical diameter of clumps sizes in DYNAMO galaxies, when measured in the $z \sim 0.1$ resolution maps is $\left\langle d_{\text {clump }}>\sim\right.$ $0.6 \mathrm{kpc}$. When making the same measurement of the $\mathrm{H} \alpha$ maps that have been blurred to match $z \sim 2$ the typical size increases to $<d_{\text {clump }}>\sim 2.2 \mathrm{kpc}$. This is just over a factor of $2 \times$ increase in clump sizes. In the $z \sim 2$ blurred maps only $\sim 44 \%$ of the clumps that are identified at $z \sim 0.1$ resolution are detected. The other clumps are either undetectable due to lower surface brightness or blend into a single clump are lower resolution.

Perhaps the most critical similarity of DYNAMO galaxies to high redshift star forming galaxies is that both are quite gas rich and very actively forming stars. Using star formation rates derived from integral field spectroscopic maps Green et al. (2014) estimates the star formation rates of DYNAMO galaxies range from $\sim 1-80 \mathrm{M}_{\odot} \mathrm{yr}^{-1}$, and from Sloan Digital Sky photometry we determine stellar masses of $\sim 0.1-10 \times 10^{10} \mathrm{M}_{\odot}$ for the same galaxies. Fisher et al. (2014) finds that DYNAMO galaxies have molecular gas fractions, $f_{m o l}=M_{m o l} /\left(M_{m o l}+M_{\text {star }}\right)=20-35 \%$. In Fig. 3 (left) we compare the gas fractions of 3 DYNAMO galaxies to that of 3 surveys of galaxies. The gas fractions of DYNAMO galaxies are found to be extreme outliers compared to local galaxies of the COLD GASS survey (Saintonge et al. 2011). The gas fractions of DYNAMO galaxies are very similar to what is observed in high- $z$ samples (eg. PHIBSS Tacconi et al. 2013). 

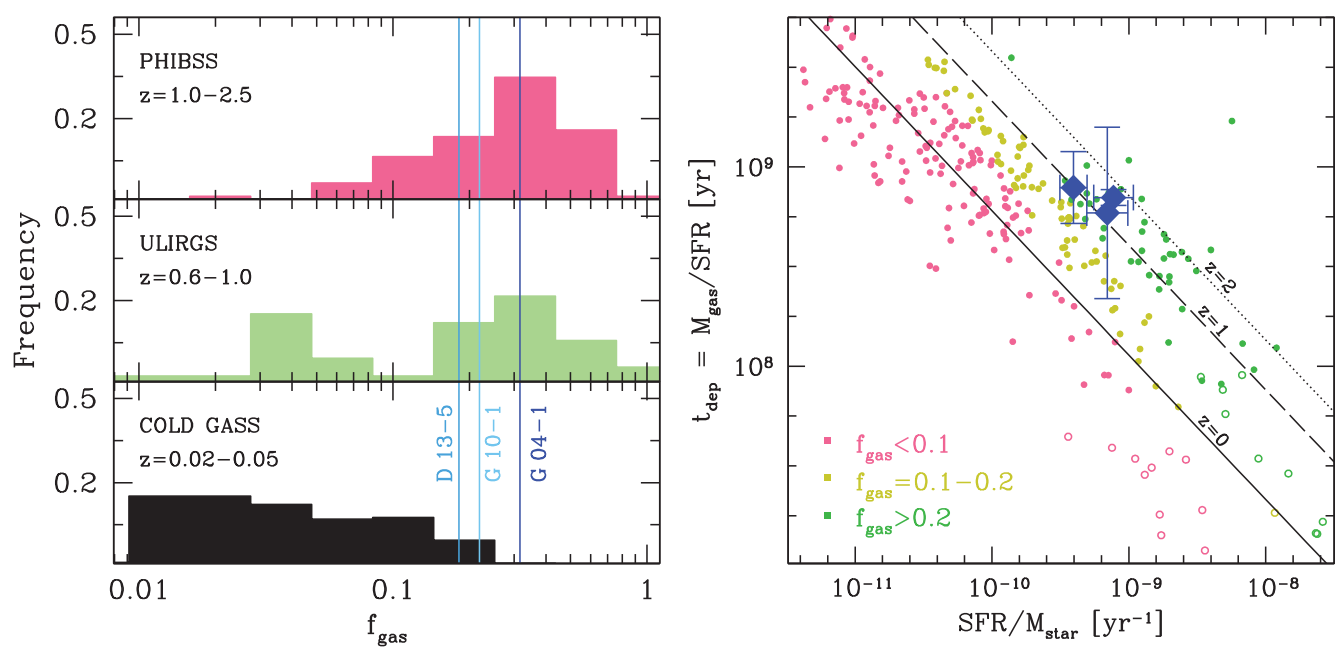

Figure 3. Both panels are replotted from Fisher et al. (2014). Left shows molecular gas fractions of three galaxies from the DYNAMO survey are compared to the distribution of gas fractions from 3 surveys. From bottom to top, those surveys of molecular gas are the $\mathrm{z}=0$ COLD GASS survey (Saintonge et al. 2011), the survey of intermediate redshift ULIRGS (Combes et al. 2013), and the PHIBSS survey of $z \sim 1-3$ galaxies (Tacconi et al. 2013). The gas fractions of DYNAMO galaxies (vertical lines) are more consistent with high redshift galaxies, than those of the low-Z Universe. Right shows the relationship between gas depletion time $\left(t_{d e p}=M_{m o l} / S F R\right)$ and specific star formation rate $\left(S F R / M_{\star}\right)$. The data is taken from the same samples as in left panel. The points are colored by gas fractions, as indicated by the key. The DYNAMO galaxies are forming stars in a manner consistent with their gas fractions.

Furthermore, DYNAMO galaxies are forming stars appropriately for their gas fraction, not their redshift. Galaxies with large specific star formation rates are known to have more efficient star formation (ie. shorter gas depletion timescales, Saintonge et al. 2011). However, galaxies at higher redshift, with larger gas fractions, have longer gas depletion times than a galaxy with the same specific star formation rate in the nearby Universe. Fisher et al. (2014) finds that DYNAMO galaxies have $t_{d e p} \sim 0.5$ Gyr. Again they are found to be more similar to galaxies of the high- $z$ Universe than nearby galaxies.

\section{References}

Abraham, et al. 1996, ApJS, 107, 1

Bassett, et al. 2014, MNRAS, 442, 3206

Combes, et al. 2013, A\& $A, 550$, A41

Elmegreen, B. G., Bournaud, F., \& Elmegreen, D. M. 2008, ApJ, 688, 67

Fisher, et al. 2014, ApJ, 790, L30

Genzel, et al. 2014, ApJ, 785, 75

Genzel, et al. 2011, ApJ, 733, 101

Green, et al. 2010, Nature, 467, 684

Green, et al., P. J. 2014, MNRAS, 437, 1070

Jones, et al. 2010, MNRAS, 404, 1247

Saintonge, et al. 2011, MNRAS, 415, 61

Swinbank, et al. 2012, ApJ, 760, 130

Tacconi, et al., 2013, ApJ, 768, 74

Wisnioski, et al., C. 2012, MNRAS, 422, 3339 